Int. J. Electrochem. Sci., 13 (2018) 1013 - 1026

\title{
Synthesis, Characterization and Electrochemical Evaluation of Polyvinylalchol/Graphene Oxide/Silver Nanocomposites for Glucose Biosensing Application
}

\author{
Seyed Morteza Naghib ${ }^{1, *}$, Ehsan Parnian ${ }^{2}$, Hamid Keshvari ${ }^{2}$, Eskandar Omidinia ${ }^{3, *}$, \\ Mahdi Eshghan-Malek ${ }^{1}$ \\ ${ }^{1}$ Nanothechnology Department, School of New Technologies, Iran University of Science and \\ Technology (IUST), P.O. Box 16846-13114, Tehran, Iran. \\ ${ }^{2}$ Department of Biomedical Engineering, Amirkabir University of Technology (Tehran \\ Polytechnique), P.O. Box 15875-4413, Tehran, Iran. \\ ${ }^{3}$ Enzyme Technology Lab., Genetics \& Metabolism Research Group, Pasteur Institute of Iran, P.O. \\ Box 13164, Tehran, Iran. \\ *E-mail: naghib@iust.ac.ir, skandar@pasteur.ac.ir
}

doi: $10.20964 / 2018.01 .74$

Received: 26 September 2017 / Accepted: 20 November 2017 / Published: 16 December 2017

\begin{abstract}
Hybridization of nanoscale metals and carbon nanostructures into composite nanomaterials has produced some of the best-performing super capacitors and biosensors to date. The challenge remains to develop scalable nanofabrication methods that are amenable to the development of biosensors. A scalable nanostructured composite based on graphene oxide nanosheets (GO), Ag nanoparticles, and a biorecognition element (glucose oxidase) was presented. In addition to good conductivity and biocompatibility, this matrix had a low total cost. The surface of composite was rough due to the presence of GO in PVA matrix that could increase contact area between composite and enzyme layer and consequently enhanced biosensor electrochemical response. Surface morphology was analyzed by SEM and characterization of the composite elements was carried out using FTIR and XRD. Finally, impedance of different samples was determined by electrochemical impedance spectroscopy (EIS) and the effect of GO amount on impedance of the composite was investigated. This method to synthesis of the nanocomposite was devoted to glucose sensing succeeded a favorable device design that also exhibited remarkable glucose biosensing performance such as improved glucose sensitivity ( $25 \mathrm{nM}$ limit of detection, $15.32 \mathrm{mAM}^{-1} \mathrm{~cm}^{-2}$ of sensitivity, $150 \mathrm{nM}$ to $10 \mathrm{mM}$ of linear range), and excellent selectivity. To the best our knowledge, this device is the first biosensor protocol that used the nanocomposite for glucose determination.
\end{abstract}

Keywords: nanocomposite, graphene oxide, polyvinylalchol, biorecognition element, FTIR, XRD

\section{FULL TEXT}


(C) 2018 The Authors. Published by ESG (www.electrochemsci.org). This article is an open access article distributed under the terms and conditions of the Creative Commons Attribution license (http://creativecommons.org/licenses/by/4.0/). 